\title{
ANÁLISE QUALITATIVA DAS PUBLICAÇÕES NACIONAIS E INTERNACIONAIS EM ETNOGRAFIAS EM ADMINISTRAÇÃO E ESTUDOS ORGANIZACIONAIS
}

\author{
QUALITATIVE ANALYSIS OF NATIONAL AND \\ INTERNATIONAL ETHNOGRAPHIC PUBLICATIONS IN MANAGEMENT AND \\ ORGANIZATIONAL STUDIES
}
Recebido em: 26/08/2012 Aprovado em: 21/01/2013
Avaliado pelo sistema double blind review
Editora Científica: Manolita Correia Lima

\author{
CHEN YEN-TSANG yentsang.chen@gmail.com \\ RONALDO GOMES DULTRA-DE-LIMA \\ KARINA PRETTO \\ FUNDAÇÃO GETÚLIO VARGAS - ESCOLA DE ADMINISTRAÇÃO DE EMPRESAS DE SÃO PAULO
}

\begin{abstract}
RESUMO
A proposta deste artigo é investigar a evolução intelectual da etnografia nas áreas de administração e estudos organizacionais no período de 1995 a 20II, por meio da análise bibliométrica de citação e cocitação em publicações internacionais e, a partir de 2000 , em publicações nacionais. Em relação às publicações nacionais, à medida que não é possível empregar a mesma técnica de citação e cocitação, optou-se por uma análise mais contextual do estágio em que se encontra a etnografia no Brasil. A metodologia empregada na condução desta pesquisa foi uma análise documental do estágio da etnografia em publicações internacionais e nacionais. Observou-se que, em estudos internacionais, os trabalhos etnográficos de Geertz (1973), Van Maanen (1988) e Clifford e Marcus (1986) tiveram contribuições importantes e, portanto, influenciaram sobremaneira as pesquisas posteriores. Essas pesquisas mostraram que esses autores tiveram maior proximidade e formaram o principal grupo em relação ao tema central. Também não foram observadas grandes alterações em relação ao cluster central, no período analisado, em periódicos internacionais. No âmbito nacional, pode-se afirmar que os estudos etnográficos ainda apresentam baixa popularidade entre os acadêmicos da área de administração, possivelmente, devido ao próprio método de pesquisa que requer longos períodos de tempo desde a coleta de dados, análise e até a publicação final.
\end{abstract}

Palavras-chaves: pesquisa qualitativa; etnografia; análise bibliométrica; cocitação; análise de citação.

ABSTRACT

The purpose of this paper is to investigate ethnography's intellectual evolution in the fields of management and organizational studies, for the period 1995 to 2011, by analyzing citations and co-citations in international publications, and in national ones from 2000 to now. As it is not possible to employ the same citation and co-citation analysis technique for the national publications, a contextual analysis of the stage Ethnography is found in Brazil was opted for. The methodology used in the research was a documentary analysis of the stage of ethnography in international and national publications. It was noted that for international studies, the ethnographic work of Geertz (1973), Van Maanen (1988) and Clifford and Marcus (1986) made important contributions, thus greatly influencing subsequent research. This research has shown that these authors, as regards the central theme, had greater proximity to it and formed its main group. Major changes were also observed in relation to the central cluster for the period examined regarding international journals. At the national level, it can be affirmed that ethnographic studies still has low popularity among academics in administration, possibly due to that the fact it requires long periods of time from the data collection, through analysis and until final publication.

Keywords: qualitative research; ethnography; bibliometric analysis; co-citation; citation analysis. 


\section{INTRODUÇÃO}

Como evoluiu a pesquisa qualitativa fundamentada nos moldes etnográficos nas pesquisas nacionais e internacionais considerando as áreas de administração e estudos organizacionais?

Diferentemente da pesquisa quantitativa, que utiliza métodos estatísticos para emitir um parecer acerca do objeto em estudo e que, em determinada situação, não consegue desvendar todo o processo de construção da realidade, a pesquisa qualitativa vai além à medida que conta com diversas técnicas disponíveis para a coleta e análise de dados. Além disso, um ponto importante diz respeito à percepção e sensibilidade do pesquisador no processo de análise que, no caso da pesquisa quantitativa, se dá por meio do estudo das relações entre as variáveis. Essa percepção e a sensibilidade do pesquisador que, de certa forma, representam uma vantagem da pesquisa qualitativa em relação à quantitativa, é bastante criticada por esta última. No entanto, no contexto qualitativo, a percepção e a sensibilidade são inerentes ao processo de entendimento dos múltiplos aspectos da realidade e permitem uma interpretação própria do fenômeno e não há como segregá-las.

Segundo Godoy (1995a), a pesquisa qualitativa tem como preocupação fundamental o estudo e a análise do mundo empírico em seu ambiente natural. Nessa abordagem, valoriza-se o contato direto e prolongado do pesquisador com o ambiente e a situação em estudo. Historicamente, essa metodologia tem origem em meados do século xix, porém, foi no século $\mathrm{XX}$ que ganhou espaço entre os acadêmicos, principalmente, no campo da psicologia e das ciências sociais. O trabalho pioneiro de Malinowski (1916), no início desse século, marca o processo de evolução dessa metodologia. A Escola de Chicago, fundada por sociólogos americanos, também teve uma papel importante no processo de evolução da pesquisa qualitativa, principalmente, a partir da década de 1930. No entanto, segundo Flick (2009), a partir da década de 1970, há uma redescoberta da pesquisa qualitativa, principalmente no campo das ciências sociais e da psicologia, em decorrência do desenvolvimento de novos métodos originais de pesquisa que se 
estendeu até meados dos anos I980, por exemplo, a entrevista narrativa e hermenêutica objetiva.

No processo de investigação em estudos qualitativos, Godoy (I995b) afirma que este pode ser conduzido por diferentes caminhos, porém, os tipos mais conhecidos e utilizados pelos acadêmicos são: o estudo de casos, a pesquisa documental e a etnografia.

Yin (2002) afirma que o estudo de caso corresponde a uma forma diferenciada de investigação empírica. Para Godoy (1995b), o estudo de caso caracteriza-se como um tipo de pesquisa cujo objeto é uma unidade que se analisa profundamente. Em ambientes organizacionais, essa metodologia tem sido bastante utilizada pela área de marketing.

A pesquisa documental tem como foco a análise de materiais escritos e, nesse caso, podem ser citados, por exemplo: jornais, revistas, diários, obras literárias, científicas e técnicas, cartas, memorandos, relatórios, etc. (GODOY, 1995b).

A etnografia, também analisada por Godoy (1995b), consiste em uma abordagem metodológica que visa o entendimento dos eventos e processo sociais e teve como marco de sua evolução a partir da década de I980, principalmente, no campo ciências sociais (FLICK, 2009). Essa abordagem corresponde a uma forma de pesquisa, que estava associada anteriormente à antropologia e que mais tarde foi adotada por outras áreas das ciências sociais, por exemplo, a educação, a psicologia social e a administração de empresas (GODOY, I995b). Conceitualmente, a etnografia pode ser entendida como a arte e a ciência de descrever e interpretar uma cultura ou grupo (GODOY, I995b), porém, não há unanimidade nesta definição. Watson (20II), por exemplo, argumenta que a etnografia não representa um método de pesquisa, mas sim um produto desta. Van Maanen (2006), analisando os estudos etnográficos nos últimos 20 anos, argumenta que o desenvolvimento dessa metodologia se deve aos múltiplos locais de pesquisa. Também observou que os trabalhos realizados nesse período foram essencialmente empíricos.

Este artigo tem como objetivo tentar responder à questão formulada no início desta introdução, por meio de uma análise bibliométrica dos 
artigos que foram publicados de 1995 a 201 s sobre a etnografia, visando identificar quais os trabalhos mais relevantes que suportaram as pesquisas neste período, tanto do ponto de vista internacional quanto nacional. Esse estudo será analisado por meio da técnica de citação e cocitação, que visa à mensuração da produção científica em uma determinada área de interesse.

Para orientar e expandir a discussão do presente trabalho, nas seções seguintes apresenta-se o referencial teórico, bem como a definição da pesquisa e os métodos utilizados para o estudo da evolução da pesquisa qualitativa no cenário internacional e nacional. Por fim, apresentam-se a discussão dos resultados, as percepções dos autores sobre os dados coletados e suas considerações finais. 


\section{REFERENCIAL TEÓRICO}

\section{SOBRE A PESQUISA QUALITATIVA}

De acordo com Yin (2002), a popularidade do estudo de caso em pesquisas empíricas muitas vezes tem causado a errônea impressão de que a pesquisa qualitativa e o estudo de caso são sinônimos. Segundo Godoy (I995a) e Denzin e Lincoln (I994), a pesquisa qualitativa não se resume ao estudo de caso, ela corresponde a uma estratégia de pesquisa que abrange diversos métodos, orientações teóricas, perspectivas e paradigmas tais como positivista, pós-positivista, construcionista e paradigma crítico. Esses pressupostos da pesquisa qualitativa fazem com que a possibilidade de sua definição metodológica possa variar de diretrizes investigativas rigorosas e estruturadas às formas menos estruturadas e mais flexíveis.

A pesquisa qualitativa, antes de ser amplamente adotada no campo de ciências sociais aplicadas, teve origem em meados do século xıx, na antropologia e sociologia. Segundo Godoy (1995a) e Bluhm et al., (2010), a introdução dessa estratégia de pesquisa nessas áreas se deu por volta da década de 1970 e, sob esta perspectiva, pesquisas em fenômenos organizacionais diversificaram os métodos de trabalho, técnicas e estilos de análise e apresentação dos resultados.

De acordo com Bluhm et al. (2010) e Godoy (1995a), a pesquisa qualitativa apresenta, basicamente, quatro características essenciais: a) o ambiente natural como fonte direta de dados e o pesquisador como instrumento fundamental; b) o caráter descritivo; c) o significado que as pessoas dão às coisas e à sua vida como preocupação do investigador; e d) enfoque indutivo na análise de seus dados. Essas quatro características poderiam ser consideradas inquestionáveis há alguns anos atrás, entretanto, o crescimento da influência tecnológica, assim como o surgimento das comunidades virtuais, possibilitou novas oportunidades quanto ao ambiente de pesquisa, como mencionado por Williams (2007).

Ao comparar a publicação de Godoy (1995b) e Denzin e Lincoln (2005), nota-se que as formas de pesquisa qualitativa podem se resumir em três 
principais abordagens: a) pesquisa documental; b) estudos de caso (incluindo a teoria fundamentada - grounded theory); e c) a etnografia. Cada uma dessas abordagens parte de pressupostos filosóficos diferentes e deve empregar técnicas de análises diferentes, uma vez que não há uma única forma de análise na pesquisa qualitativa. Além disso, alinhado às recomendações de Denzin e Lincoln (1994), Bluhm et al. (2010) também concordam que cada análise deve se ajustar de forma única ao fenômeno observado.

Considera-se que a análise de dados, segundo Strauss (2003), representa o sinônimo de interpretação, podendo ocorrer nos diversos níveis de explicitação, abstração e sistematização. No nível mínimo de abstração, estão as descrições; no mais alto, os pesquisadores estão focados na geração de teoria.

\section{SOBRE A ETNOGRAFIA}

Nas três abordagens da pesquisa qualitativa levantadas anteriormente, é injusto afirmar que uma abordagem é mais importante do que outra, já que, conforme Yin (2002) e Denzin e Lincoln (1994), a estratégia de pesquisa depende do fenômeno a ser investigado e da pergunta da pesquisa. Nesse caso, haverá sempre uma abordagem que seja mais adequada a um determinado contexto.

Duas das três perspectivas levantadas - a pesquisa documental e o estudo de caso - são amplamente conhecidas em pesquisas das ciências sociais aplicadas. A primeira, é empregada na área mercadológica e visa analisar os conteúdos veiculados nas propagandas e entender a complexidade do ambiente multicultural; a segunda, aplica-se, por exemplo, em diversas investigações de processos de inovação nas empresas multinacionais (AGUIAR, 2004; GODOY, 1995C). A terceira abordagem da pesquisa qualitativa referese à etnografia. Essa é uma forma de pesquisa associada à antropologia e, segundo Godoy (1995b), tem sido continuamente adotada por outras áreas das ciências. Para Godoy (1995b), a etnografia na sua concepção mais ampla é a arte e a ciência de descrever uma cultura ou grupo. Já Watson (20II), de forma mais pragmática, afirma que etnografia não é um método de pesquisa, mas sim o produto da pesquisa. Esse autor ainda argumenta 
que a etnografia é um estilo de escrita da ciência social, que se baseia na estreita observação do escritor e do seu envolvimento com pessoas em uma determinada configuração social. Nesse processo, o investigador deve relacionar as palavras ou falas capturadas no ambiente às práticas observadas dentro do contexto no qual a dinâmica ocorre.

Sob a luz desta abordagem, Godoy (1995b), Van Maanen (2006, 20II) e Watson (20II) argumentam que o investigador etnográfico necessita ter contato estreito com o objeto investigado, na sua forma natural, e por um longo período de tempo suficiente para apreciar as normas formais, informais, valores e práticas inerentes ao meio. Ao longo deste processo, o investigador deve superar as barreiras a ele impostas e empregar métodos adequados de coleta de dados, tais como observação, participação nas atividades cotidianas, entrevistas e coleta de documentos.

Assim como em outras abordagens qualitativas, a etnografia exige uma profunda reflexão e interpretação por parte do investigador na análise de dados e, muitas vezes, para que isso ocorra, o investigador necessita se retirar do ambiente pesquisado. Como experiências prévias citadas estão aquelas mencionadas por Kunda (2006), em seu livro Engineering Culture: Control and Commitment in a Hight Tech Corporation, e Whyte (I943), em seu livro Street Corner Society: The Social Structure of an Italian Slum.

A etnografia, assim como outras estratégias de pesquisa, tem evoluído ao longo dos anos e de certa forma se ajusta ao contexto no qual um pesquisador a emprega. Diante disso, Van Maanen (2006) discorre sobre a evolução da etnografia e aborda, por exemplo, a questão de múltiplos locais de pesquisa, a questão relacionada ao tempo e espaço e à mudança cultural dos leitores dos trabalhos. Apesar dessas mudanças, segundo esse autor, a etnografia na sua essência ainda continua sendo a mesma desde o lançamento do livro Tales of the Field: On Writing Ethnography, de Van Maanen (I988).

\section{SOBRE O MÉTODO DE PESQUISA}

Conforme abordado anteriormente, uma das formas da pesquisa qualitativa envolve a análise documental. Segundo Godoy (I995b), sob essa perspectiva, 
os documentos a serem examinados podem ser: jornais, revistas, diários, obras literárias, científicas e técnicas, cartas, memorandos, as estatísticas (que reproduzem um registro ordenado e regular do objeto estudado) e elementos iconográficos. Essa forma de pesquisa possui algumas vantagens; por exemplo, permite o estudo de objetos, aos quais o pesquisador não tem acesso; além disso, permite ao pesquisador estudar longos períodos de tempo, buscando identificar uma ou mais tendências no comportamento do objeto investigado (GODOY, 1995b). Sob essa ótica, a pesquisa da literatura pode ser considerada uma pesquisa documental em que os investigadores visam identificar quais são os temas centrais na área de interesse e quais seriam as tendências e as necessidades de pesquisa da comunidade científica.

Os pesquisadores Usdisken e Pasadeos (1995) citaram algumas formas de pesquisa referentes à literatura de uma área de estudos, entre as quais é possível destacar: a) a revisão de literatura, cuja natureza é geralmente qualitativa e tenta estabelecer os princípios e paradigmas encontrados em uma grande quantidade de estudos na área; b) a meta-análise, que em sua natureza quantitativa, procura agregar os resultados obtidos em vários estudos na área; c) a investigação metodológica que avalia os métodos de pesquisa conduzidos nos estudos; d) estudos de produtividade de publicação que focam basicamente nos autores e nas instituições e como esses contribuem para a literatura; e) estudos de periódicos específicos que são caracterizados por uma investigação em profundidade de um ou mais aspectos das publicações; e f) os estudos de citação, que investigam as referências bibliográficas dos trabalhos acadêmicos.

Back (1974) afirma que os estudos de citação são uma forma de investigação fundamentada nas referências bibliográficas que representam uma forma de expressão aparente da comunicação científica e retratam o conhecimento e as atividades do campo de estudos, sendo capazes de denotar padrões sociológicos e históricos. Considerando as informações explícitas e implícitas nas referências bibliográficas, as análises bibliométricas utilizam muitas vezes técnicas de citação e cocitação, visando à mensuração da produção científica no sentido de avaliar esse conhecimento e essas atividades do campo de estudos. 
De acordo com Small (1973), a cocitação pode ser definida como a frequência com a qual dois trabalhos prévios de uma determinada área literária são citados conjuntamente por trabalhos posteriores. A "força" da cocitação é medida com base no nível de relacionamento ou na associação entre artigos, uma vez estabelecida a população de autores citados. $\mathrm{O}$ resultado das cocitações mede a produção de pesquisa científica e tecnológica, na forma de artigos, publicações, citações, patentes e outros indicadores mais complexos. Adicionalmente, os padrões de cocitação se alteram à medida que os interesses e padrões intelectuais de uma determinada área mudam. Pode-se assumir que artigos frequentemente citados representam os conceitos, métodos ou experimentos chaves de uma área de pesquisa. Nesse sentido, os padrões de cocitações podem ser utilizados para mapear, em detalhe, os relacionamentos entre estas ideias, ou seja, trata-se de um modo objetivo de modelar a estrutura intelectual de determinado campo científico.

Vale ressaltar que nas análises bibliométricas utilizam-se apenas a comunicação formal entre os cientistas: artigos; livros; patentes; documentos; e não são consideradas as comunicações informais (literatura cinzenta), tais como: oral; relatórios; conferências; comunicação eletrônica não científica. Ainda sobre a análise bibliométrica, Lim et al. (2009) avaliam a qualidade da citação e apontam para o viés de que a quantidade de vezes que um autor é citado não necessariamente indica a qualidade da obra. Para eles, a publicação de uma citação em periódico de prestígio pode superar uma citação publicada em periódico que não tenha o mesmo reconhecimento. E apontam que a qualidade da citação pode ser indicada mediante quão importante e relevante é o periódico em que a citação foi publicada na área de interesse. Seglen (1992) também aborda a questão da qualidade em citações e afirma que as citações correspondem a uma medida de impacto da influência global do artigo ou de seus autores, em uma determinada comunidade científica, o que induziria a um fator de qualidade; entretanto, este fator não é equivalente e nem pode ser correlacionado com a qualidade científica. Apesar de suas limitações, a bibliometria proporciona uma medida essencialmente objetiva da produção científica (ОкUво, 1997). 
No Brasil, Balestrin, Verschoore e Reyes Jr. (2010), avaliando os estudos sobre redes de cooperação interorganizacionais por meio da análise bibliométrica por redes sociais, destacaram que das oito universidades brasileiras que mais contribuíram para a pesquisa no país, levando em consideração os aspectos quantitativos de publicações, três instituições (UFRGS, UFRJ e UNISINOS) apresentaram elevada centralidade que, segundo os autores, indica que as publicações são realizadas em conjunto com autores de outras universidades. 


\section{DEFINIÇÃO DA PESQUISA E MÉTODOS}

Neste trabalho, pretende-se avaliar, de forma qualitativa, a produção etnográfica nacional e internacional publicada no campo da administração e estudos organizacionais. A seleção dos periódicos foi baseada na sua relevância acadêmica dada por seu fator de impacto pelo Journal Citation Reports/2011 (JCR) à área de interesse desta pesquisa e que deveria estar disponível para consulta nas principais bases de dados de artigos. Os periódicos internacionais selecionados cujos fatores de impactos estão entre parênteses foram: Gender, Work and Organization (I,206); Human Relations (I,729); Journal of Advertising Research (I,400); Journal of Business Research (I,872); Journal of Consumer Research (3,100); Journal of Information Technology (2,321); Journal of Management Studies (4,255); Journal of Organizational Change Management (0,744); Organization (I,67I); Organization Studies (2,328); Organizational Research Methods $(3,257)$. Para se ter uma ideia da importância desses periódicos, uma das principais revistas brasileira em administração (Revista de Administração de Empresas - RAE - da FGV/EAESP) possui fator de impacto de 0,213 pelo referido periódico. Sendo assim, duas bases de artigos científicos foram consideradas representativas das publicações internacionais e nacionais: a ISI Web of Knowledge e Scientific electronic Library Online (Scielo), respectivamente.

O critério de busca dos trabalhos científicos nacionais (base: Scielo) contém três dimensões: ano; periódico; e palavras-chave da publicação. Faz parte deste estudo toda a produção acadêmica nacional que possui a palavra etnografia entre suas palavras-chave e cuja data de publicação seja igual ou posterior ao ano 2000. A seleção dos periódicos foi baseada na sua relevância para a pesquisa em administração de empresas. Foram selecionados: Revista de Administração de Empresas (RAE) e Revista de Administração de Empresas Eletrônica (RAE eletrônica), Revista de Administração Contemporânea (RAC), Revista Organização e Sociedade (o\&s) e Cadernos eвApe. Estes dois últimos foram incluídos na pesquisa devido à sua reconhecida contribuição à produção acadêmica em pesquisas em administração de empresas ou áreas afins. Foram consultados todos os 
seus artigos e selecionados não só pelas palavras-chave, mas pelo fato de o artigo ser decorrente de temas relacionados a estudos etnográficos.

Ao final deste processo, quinze artigos nacionais satisfizeram os critérios acima citados e seguiram para a avaliação qualitativa dos temas abordados por meio da leitura destes trabalhos. Todos os trabalhos foram lidos e classificados quanto ao tema, forma da pesquisa e ano de publicação. Dessa forma, é possível explorar os aspectos metodológicos mais comuns entre os estudos etnográficos brasileiros e fazer uma avaliação sobre a evolução do tema na última década.

O protocolo da pesquisa, detalhado a seguir e conforme mostra a Figura I, foi aplicado à base de periódicos da ISI Web of Science (periódicos internacionais) e no período compreendido entre os anos de 1995 a 201 I. A escolha dos periódicos foi definida pela quantidade de artigos publicados no período estabelecido sobre o tópico etnografia, bem como pela importância do mesmo. Dessa forma, onze periódicos científicos participaram do estudo, conforme apontado acima.

Com base na seleção dos artigos, avaliam-se as citações e cocitações, a fim de identificar o padrão de associação entre os autores. Para tal, os dados foram coletados na base de dados ISI Web of Science e Scielo para as revistas brasileiras. 
IDENTIFICAÇÃO DOS ARTIGOS

\section{SELEÇÃO DE ARTIGOS INTERNACIONAIS}

Tendo em vista o método de pesquisa adotado - pesquisa documental - e do objetivo, a presente pesquisa utilizou a palavra chave "ethnography" para buscar artigos científicos internacionais, que abordam este tópico na base de dados da ISI Web of Knowledge (Web of Science), considerando o período de 1995 a 20II. A escolha desse período teve como ponto fundamental a consolidação da pesquisa qualitativa a partir dos anos de 1980 na área da administração (BLUHM et al., 20I0; GODOY, I995a), e, mais especificamente, a etnografia baseada na publicação do livro de Van Mannen (1988) - Tales of the Field: On Writing Ethnography.

Cabe enfatizar que na proposição da pesquisa foi delimitada a área da Administração (Management) e Estudos Organizacionais como o foco deste trabalho, na busca e análise de artigos científicos. Também foram selecionados onze periódicos internacionais que mais publicaram artigos relacionados ao tópico etnografia nos últimos i5 anos e que, entre os quais, estão os melhores em estudos organizacionais.

\section{SELEÇÃO DE ARTIGOS NACIONAIS}

Para comparar a evolução dos estudos etnográficos do cenário nacional com o internacional foram escolhidos artigos científicos publicados nas principais revistas nacionais: Revista de Administração de Empresas (RAE) e Revista de Administração de Empresas Eletrônica (RAE eletrônica); Revista de Administração Contemporânea (RAC); Revista Organização e Sociedade (O\&s); e Cadernos EBAPE.

\section{APLICATIVOS DE AUXÍLIO AO GERENCIAMENTO DE DADOS E SUPORTE À ANÁLISE}

De acordo com Bandeira-de-Melo (2006) e Weitzman (2000), o uso de programas de auxílio à pesquisa qualitativa traz benefícios tais como organização, economia de tempo, rigor, transparência e novas possibilidades 
de tratamento de dados e análises. Tendo em vista esses benefícios, nesta pesquisa, devido ao grande volume de dados a serem gerenciados (centenas de artigos e milhares de citações), decidiu-se pela adoção dos softwares Sitkis e Ucinet para auxiliar nas tarefas de gestão de base de dados e suporte à análise dos resultados. O primeiro software está escrito em linguagem Java, e importa registros da base de dados ISI Web of Science, criando uma base de dados no aplicativo Microsoft Access $\mathbb{B}$ que pode ser facilmente manipulada (SCHILDT, 2002).

Figura I Etapas da Metodologia

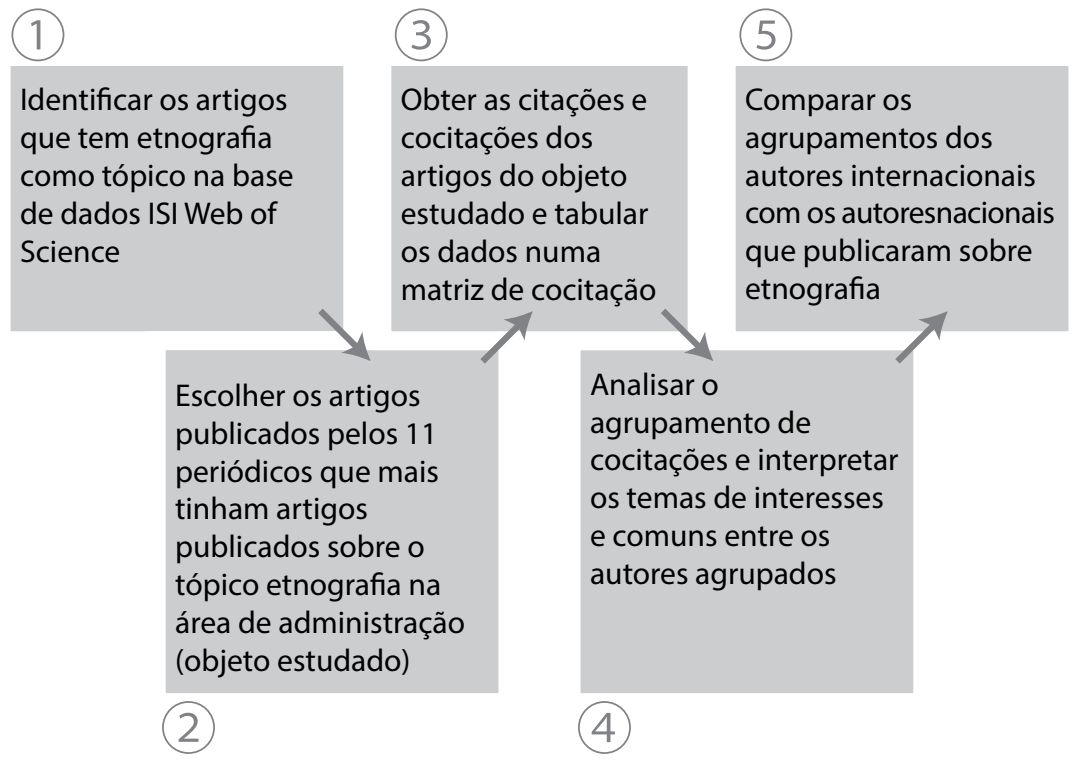

O conceito deste software trata cada artigo selecionado com base no ISI Web of Science como um caso a ser analisado e auxilia na criação de tabulações de citação e cocitação para análise. O princípio de emprego de software de auxílio à pesquisa qualitativa é análogo aos tradicionais softwares como: Nvivo, Atlas.ti ou QDA Miner usados, por exemplo, em processos de codificação de documentos e imagens. Entretanto, o Sitkis está voltado à finalidade de análise bibliométrica. Adicionalmente, também foi utilizado 
o aplicativo UCINET destinado à análise de redes sociais. Com o auxílio desses softwares, os pesquisadores puderam interpretar o comportamento das cocitações e o relacionamento entre os autores (BORGATTI; EVERETT; FREEMAN, 2002).

\section{A CONSTRUÇÃO DA MATRIZ DE COCITAÇÕES}

Como já mencionado anteriormente, a cocitação é uma medida da associação entre artigos dados pela população de autores citados, pode-se assumir, desta forma, que artigos frequentemente citados representam os conceitos, métodos ou experimentos chaves de uma área de pesquisa. Nesse sentido, os padrões de cocitação podem ser utilizados para mapear, em detalhe, os relacionamentos entre estas ideias, ou seja, trata-se de um modo objetivo de modelar a estrutura intelectual de determinado campo científico.

Este estudo adotou o "artigo" como unidade de análise e objetiva revelar a "força" individual de cada um deles ao longo do tempo. Dessa forma, visando construir a matriz de cocitações foi empregada a base de dados da ISI Web of Knowledge, e o período para tratamento e análise dos dados, de 1995 a 20II, foi divido em três períodos de cinco anos cada: a) de 1995 a 2000; b) de 2001 a 2005; c) de 2006 a 20II. Na geração da matriz de cocitações, empregouse como limite de corte, artigos ou livros com, no mínimo, três citações, ou seja, para que um artigo fizesse parte da base de dados, ele deveria ter sido citado pelo menos três vezes no período por outros autores. Um exemplo da matriz de co-citações está ilustrado na Figura 2 em que as linhas estão os autores citados e na coluna estão os autores cocitados, por exemplo, na linha um e última coluna, o artigo Communication, power and organization, de Alvesson, publicado em 1996, foi cocitado três vezes com o artigo de Foucault (1980), Power/Knowledge: Selected Interviews \& Other Writings, que, por sua vez, também foi cocitado três vezes com o artigo Modernism, Post Modernism and Organizational Analysis 2: The Contribution of Michel Foucault, de Burrell (1988). 
Figura 2 Exemplo da matriz de cocitações gerada

\begin{tabular}{|c|c|c|c|c|c|c|c|c|c|c|}
\hline & & 2 & 6 & 2 & 5 & 6 & 8 & 7 & & 3 \\
\hline & & 2 & 7 & 1 & 9 & 1 & $\mathbf{0}$ & 5 & 3 & 6 \\
\hline & & AM & AM & AM & AM & AM & AE & AE & FM & FM \\
\hline 22 & ALVESSON M-COMMUNICATION POWER-1996 & 0 & 1 & 2 & 2 & 0 & 0 & 0 & 0 & 3 \\
\hline 67 & ALVESSON M-HUM RELAT-2000 & 1 & 0 & 1 & 1 & 0 & 0 & 0 & 1 & 1 \\
\hline 21 & ALVESSON M-J APPL BEHAV SCI-2000 & 2 & 1 & 0 & 2 & 0 & 0 & 0 & 0 & 3 \\
\hline 80 & ARNOULD EJ-J CONSUM RES-1993 & 0 & 0 & 0 & 0 & 0 & 0 & 0 & 0 & 0 \\
\hline 75 & ARNOULD EJ-J MARKETING RES-1994 & 0 & 0 & 0 & 0 & 0 & 0 & 0 & 0 & 0 \\
\hline 77 & BELK RW-J CONSUM RES-1989 & 0 & 0 & 0 & 0 & 0 & 4 & 3 & 0 & 0 \\
\hline 85 & BERGER PL-SOCIAL CONSTRUCTION-1966 & 0 & 1 & 1 & 1 & 0 & 0 & 0 & 0 & 1 \\
\hline 82 & BROWN JS-ORGAN SCI-1991 & 0 & 0 & 0 & 0 & 0 & 0 & 1 & 0 & 0 \\
\hline 37 & BURRELL G-ORGAN STUD-1988 & 0 & 1 & 0 & 0 & 1 & 0 & 0 & 2 & 3 \\
\hline 68 & CELSI RL-J CONSUM RES-1993 & 0 & 0 & 0 & 0 & 0 & 4 & 1 & 0 & 0 \\
\hline 46 & CLIFFORD J-WRITING CULTURE-1986 & 2 & 0 & 0 & 1 & 0 & 0 & 0 & 1 & 2 \\
\hline 17 & COOPER R-ORGAN STUD-1989 & 0 & 1 & 0 & 0 & 0 & 0 & 0 & 2 & 2 \\
\hline 72 & DENZIN NK-HDB QUALITATIVE RES-1994 & 1 & 0 & 2 & 3 & 0 & 0 & 0 & 0 & 3 \\
\hline 90 & DUGAY P-J MANAGE STUD-1992 & 0 & 0 & 0 & 0 & 0 & 0 & 0 & 1 & 1 \\
\hline 3 & FOUCAULT M-DISCIPLINE PUNISH-1977 & 0 & 1 & 0 & 0 & 0 & 0 & 0 & 0 & 3 \\
\hline 36 & FOUCAULT M-POWER KNOWLEDGE-1980 & 3 & 1 & 3 & 3 & 1 & 0 & 0 & 3 & 0 \\
\hline
\end{tabular}

\section{MÉTODO TRATAMENTO DA MATRIZ DE COCITAÇÕES}

Devido ao tamanho da matriz de cocitação, assim como sua forma de representação, não possibilita interpretações significativas sobre o cenário e as associações entre os autores internacionais. A fim de proporcionar melhor interpretação dos dados, as matrizes de cocitações geradas foram tratadas por uma técnica numérica denominada escala multidimensional, cujo conceito se baseia no cálculo da distância euclidiana em duas dimensões. Desta forma, é possível localizar os autores num plano de tal forma que as distâncias entre eles sejam compostas pelas quantidades de cocitações entre si e, consequentemente, os autores mais cocitados estarão mais próximos. 
Figura 3 Exemplo do mapa de distribuição

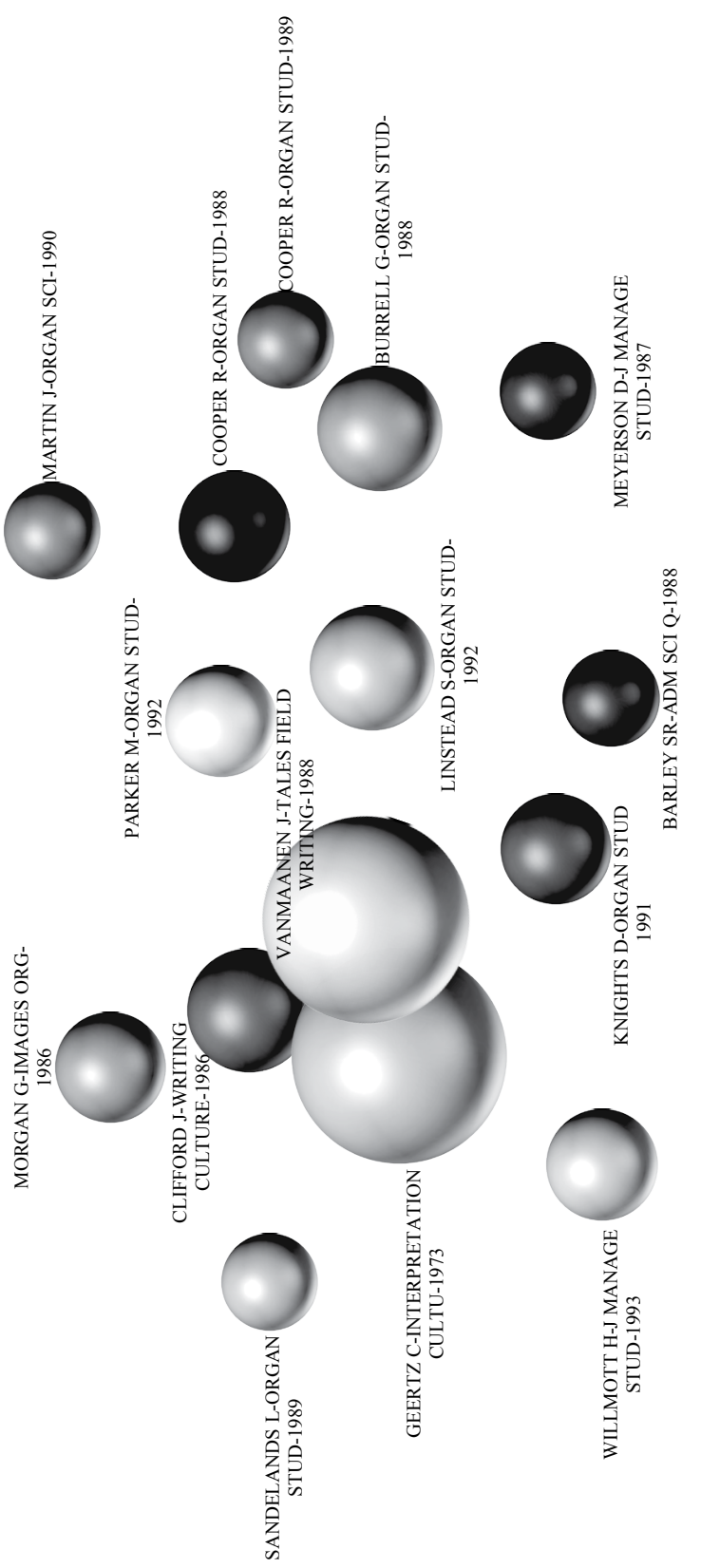


Para sua construção, a matriz de cocitações de cada período foi tratada por esta técnica o que gerou três mapas de distribuição das proximidades dos autores fundamentados nas cocitações. A esses mapas de distribuição foram adicionadas as quantidades de citações de cada autor, o que demonstra o impacto do trabalho no período estudado. Para ilustrar o conceito, a Figura 3 traz um exemplo do tratamento de uma matriz de cocitações o qual foi ajustado pelos pesquisadores e nele pode ser interpretado que, no período estudado, Geertz (1973), Van Maanen (1988) e Clifford e Marcus (1986) tiveram uma proximidade alta e dois desses três autores tiveram um impacto alto no período pesquisado (quantidade de citações). Além disso, o tema neste período esteve altamente relacionado à etnografia e, possivelmente, empregou o conceito interpretativista para as pesquisas. A seguir fez-se também uma análise por período (sendo o período I de 1995 a 2000; período 2 de 2001 a 2005; e período 3 de 2006 a 20II), visando identificar quais os trabalhos que suportaram pesquisas no âmbito da etnografia. Essa análise está baseada nas Tabelas 4, 5, e 6 . 


\section{RESULTADOS E DISCUSSÕES}

\section{TRABALHOS INTERNACIONAIS}

$\mathrm{Na}$ análise dos resultados dos artigos publicados na literatura internacional, cujo tema central refere-se à etnografia, cabe enfatizar que foram escolhidos os principais periódicos da área de administração e estudos organizacionais (vide Tabela 2). E, entre esses periódicos, para que um determinado artigo compusesse a base de dados a ser analisada deveria ter no mínimo três citações, ou seja, ter sido citado pelo menos três vezes por outros autores, ou pelo menos três cocitações, ou seja, dois autores tomados conjuntamente deveriam ter sido citados pelo menos três vezes em trabalhos de outros autores.

Com base no exposto, observa-se que na Tabela I foram publicados na literatura internacional I03 artigos até 20II cujo tema central foi etnografia. Nota-se que até 1995, apenas nove artigos (8,7\%) foram publicados, demonstrando baixo interesse por parte dos pesquisadores em relação ao tema em análise. A partir de 1995, observa-se um interesse crescente devido ao aumento de artigos publicados e parece haver evidência de que há uma mudança de perspectiva em relação aos estudos etnográficos, tanto do ponto de vista organizacional quanto do ponto de vista da cultura. Para o primeiro período, de 1995 a 2000, foram publicados 20 artigos (I9,4\%); no segundo, de 200 a 2005 , foram 23 artigos (22,3\%) publicados; e no terceiro, de 2006 a 20II, foram 5I artigos (49,5\%) publicados.

Também, analisando os principais periódicos (Tabela 2), nota-se que a amostra está distribuída entre onze periódicos e que os cinco primeiros são responsáveis por 65,0\% (67 artigos) dos artigos publicados em relação ao tema central. Os três primeiros respondem por $46,6 \%$ (48 artigos) das publicações, sendo eles: Human Relations (20,4\% com 2I artigos); Organization Studies (I4,6\% com I5 artigos); e Organization (II,7\% com I2 artigos). 
Tabela I Número de artigos publicados por período

\begin{tabular}{|c|c|c|c|}
\hline \multicolumn{4}{|c|}{ Número de artigos internacionais publicados por ano } \\
\hline Período & $N^{\circ}$ artigos & $\%$ & $\%$ acumulado \\
\hline Até 1995 & 9 & $8,7 \%$ & $8,7 \%$ \\
\hline de 1995 a 2000 & 20 & $19,4 \%$ & $28,2 \%$ \\
\hline de 2000 a 2005 & 23 & $22,3 \%$ & $50,5 \%$ \\
\hline de 2005 a 2011 & 51 & $49,5 \%$ & $100 \%$ \\
\hline Total de artigos publicados & 103 & $100 \%$ & \\
\hline
\end{tabular}

A Tabela 3 da matriz de cocitação do período de 1995 a 201 I mostra os inter-relacionamentos entre os autores e, com base nesta matriz, foram identificados quais os trabalhos que tiveram maior impacto na literatura em etnografia. A análise dessa matriz segue a seguinte lógica: os autores e trabalhos que mais foram citados conjuntamente (cocitação) têm maior impacto sobre os temas centrais da pesquisa de interesse. Por exemplo, os trabalhos de Geertz (1973) e Van Maanen (1988) aparecem com 9 citações conjuntas no período analisado. E são os autores mais citados conjuntamente, o que demostra a importância e o impacto de suas pesquisas em trabalhos posteriores. Em seu livro - The interpretation of cultures - Geertz (1973, p. 89) aborda a cultura como um sistema de concepções herdadas expressas em formas simbólicas por meio das quais as pessoas se comunicam, perpetuam e desenvolvem seu conhecimento e as atitudes diante da vida. Já Van Maanen (1988), em seu livro Tales of the Field: On Writing Ethnography explora os artifícios retóricos e narrativos usados na escrita etnográfica, tanto sociológica quanto antropológica. E divide o gênero em três categorias principais: realista, contos confessionais e impressionistas, e trata cada uma dessas categorias separadamente, ilustrando a análise com exemplos extraídos de seu trabalho de campo realizado em uma força policial dos Estados Unidos. 
Tabela 2 Número de artigos publicados por período

\begin{tabular}{|llll|}
\hline Número de publicações em periódicos internacionais & & & \\
\hline Periódico & No artigos & $\%$ & $\%$ acumulado \\
\hline Human Relations & 21 & $20,4 \%$ & $20,4 \%$ \\
\hline Organization Studies & 15 & $14,6 \%$ & $35 \%$ \\
\hline Organization & 12 & $11,7 \%$ & $46,6 \%$ \\
\hline Journal of Consumer Research & 10 & $9,7 \%$ & $56,3 \%$ \\
\hline Journal of Organizational Change Management & 9 & $8,7 \%$ & $65 \%$ \\
\hline Journal of Business Research & 8 & $7,8 \%$ & $72,8 \%$ \\
\hline Journal of Management Studies & 8 & $7,8 \%$ & $80,6 \%$ \\
\hline Gender. Work and Organization & 5 & $4,9 \%$ & $85,4 \%$ \\
\hline Journal of Advertising Research & 5 & $4,9 \%$ & $90,3 \%$ \\
\hline Journal of Information Technology & 5 & $4,9 \%$ & $95,1 \%$ \\
\hline Organizational Research Methods & 5 & $4,9 \%$ & $100 \%$ \\
\hline Total de artigos publicados & $\mathbf{1 0 3}$ & $\mathbf{1 0 0} \%$ & \\
\hline
\end{tabular}

Análise de grupo (Tabelas 4, 5 e 6) mostra claramente como estão divididos os agrupamentos de autores/trabalhos que impactaram a literatura posterior quanto ao tema em análise. Observa-se na Tabela 4, referente ao período de 1995 a 2000, que os autores mais influentes foram Geertz (1973) com o livro The interpretation of Cultures, seguido de Van Maanen (1979) com o artigo The fact of fiction in organizational ethnography, e Clifford e Marcus (1986) com Writing culture: The politics and poetics of ethnography. Também fazem parte desse grupo Rosen (1991), com o artigo Coming to terms with the field: understanding and doing organizational ethnography, e Marcus (1986), com o artigo Contemporary problems of ethnography in the modern world system. Portanto, nesse primeiro período, os trabalhos desses autores, foram os que mais impactaram nas pesquisas nos períodos seguintes. Em outras palavras, há uma alta centralidade entre esses autores oriunda do número de citações.

No segundo período, de $200 \mathrm{I}$ a 2005 , observam-se dois grupos de publicações que mais influenciaram as pesquisas no período seguinte. $\mathrm{O}$ primeiro é composto por autores e trabalhos que mais se destacaram em estudos etnográficos, em periódicos internacionais, e é liderado por Van Maanen (1988) com o livro Tales of the Field: On Writing Ethnography com 
2I citações, seguido por Geertz (1973), já abordado acima, com 20 citações. Também se observa um segundo grupo, que aparece liderado por Glaser e Strauss (1967) com o trabalho The discovery of grounded theory: strategies for qualitative research, e Shouten e McAlexander (1996) com o trabalho Subcultures of Consumption: An Ethnography of the New Bikers Subcultures of Consumption: An Ethnography of the New Bikers. O que é interessante notar é que há dois agrupamentos distintos, cujo foco das pesquisas está altamente correlacionado como tema em estudo. Então, como explicar os dois grupos? A resposta para esse fenômeno vem do fato de que se os artigos apresentarem um grande número de citações entre si ocorrerá novamente a proximidade dos autores dada pela distância euclidiana em relação ao número de citações auferidas. 

ADMINISTRAÇÃO E ESTUDOS ORGANIZACIONAIS

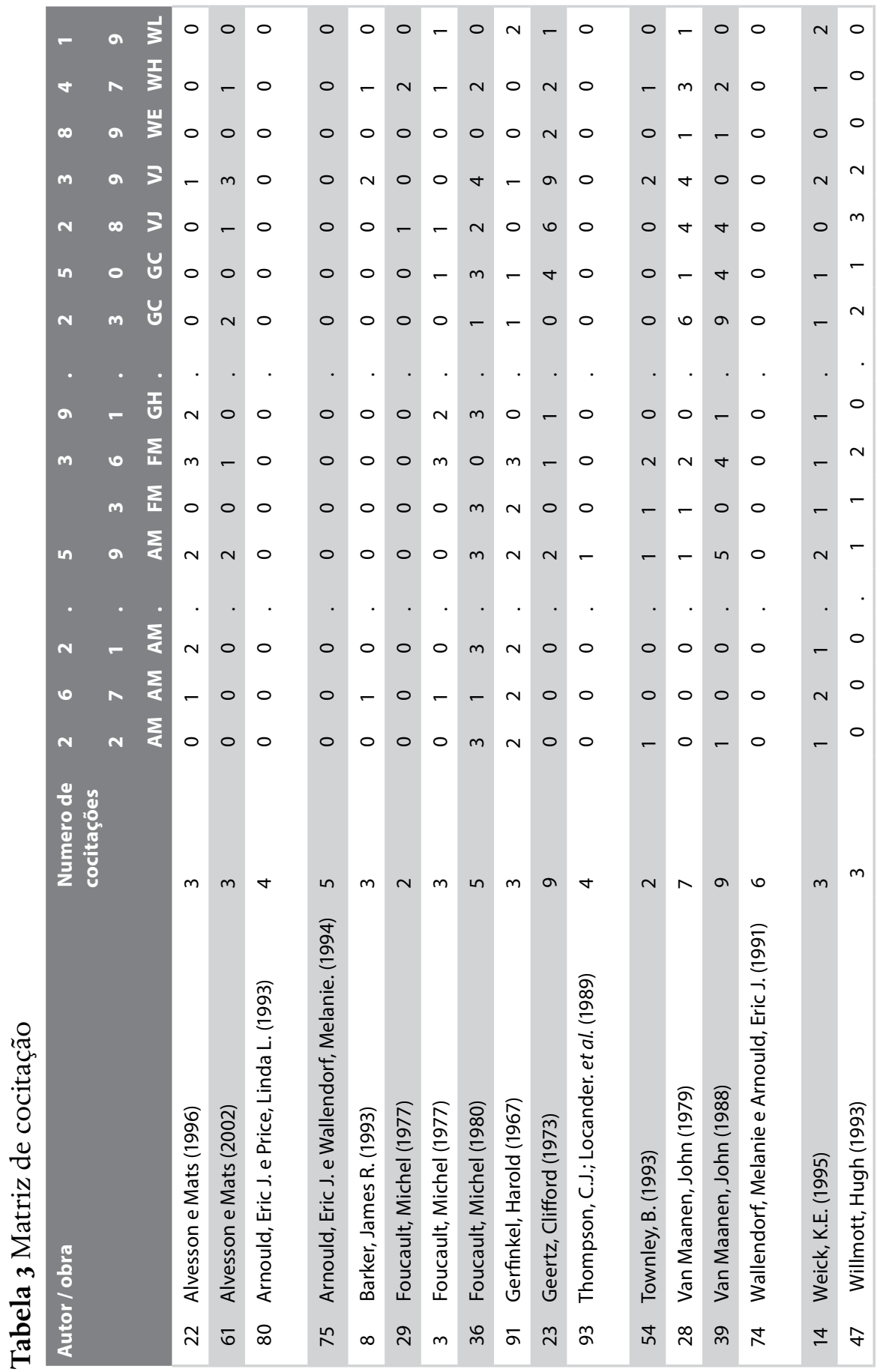


No terceiro período, de 2006 a 20II, houve pouca alteração em relação aos agrupamentos observados nos períodos anteriores. Os trabalhos que mais impactaram nas pesquisas neste período continuam sendo os trabalhos de Van Maanen (I988) com 2I citações, seguido por Geertz (I973) com 20 citações, Van Maanen (1979) com I2 citações, e Clifford e Marcus (1986), Glaser e Strauss (1967), Shouten e MacAlexander (1996) e Foucault (1980) com II citações. Esse último autor apareceu pela primeira vez na lista dos autores, cujos trabalhos se tornaram relevantes às pesquisas com o livro Power/Knowledge: Selected Interviews \& Other Writings.

Tabela 4 Grupo de artigos/autores mais citados de 1995 a 2000

\begin{tabular}{|l|}
\hline \\
\hline
\end{tabular}




\section{TRABALHOS NACIONAIS}

No âmbito nacional, os estudos etnográficos em administração de empresas são ainda bastante tímidos. Percebe-se que, no geral, somente um trabalho científico a respeito desse tema é publicado ao ano e não necessariamente são relatos etnográficos tais como descritos na literatura (GODOY, I995b; vAN MAANEN, 2006, 20II; WATSON, 20II). Os anos de 20IO e 20II aparecem com o maior número de publicações a respeito do tema, apresentando trabalhos que são frutos de estudos etnográficos, trazendo discussões metodológicas ou perspectivas sobre o uso de etnografia em pesquisas em administração de empresas. Este aumento de trabalhos publicados nos anos mais recentes pode sugerir que há esforços no meio acadêmico nacional em disseminar este tipo de abordagem metodológica.

Pode-se pensar em dois motivos para a pequena participação de estudos etnográficos na produção científica nacional em administração de empresas. O primeiro deles é que, mais frequentemente, a etnografia é utilizada e aceita em áreas como ciências sociais e antropologia. O uso dessa abordagem qualitativa em administração de empresas poderia deparar-se com dificuldades de aceitação dos periódicos e até mesmo com o desconhecimento sobre o método empregado.

Entre os dezesseis trabalhos apresentados, dois chamam a atenção pelo fato de tentarem argumentar favoravelmente ao uso da etnografia em pesquisas em administração, como um método alternativo e muito rico em informações para os pesquisadores. Em seu trabalho, Mascarenhas (2002) discute como o uso da etnografia pode auxiliar o pesquisador de administração de empresas a entender melhor a dinâmica sociocultural de uma organização, assim como os estudos antropológicos o fazem com a sociedade em geral. Por meio da comparação entre a antropologia e a administração, o autor defende o argumento de que a etnografia traz elementos que permitem o aprofundamento dos problemas enfrentados pelo administrador em seu dia a dia.

Assim como Mascarenhas (2002), Cavedon (2005) também apresenta a etnografia como uma alternativa de abordagem metodológica já conhecida em pesquisas antropológicas e com grande potencial em estudos de administração de empresas. Bem embasado teoricamente, o trabalho 
explora particularmente a possibilidade de usar fotografias para subsidiar estudos etnográficos em estudos organizacionais, denominando o método de fotoetnografia (CAVEDON, 2005).

Em 2008, Pinto e Santos (2008) discutem uma proposta sobre a conciliação entre fenomenologia, etnografia e teoria fundamentada (grounded theory), a fim de ampliar a discussão em pesquisas sobre o comportamento dos consumidores, e fomentar o debate sobre a utilização de metodologias complementares, que tragam maiores subsídios científicos aos temas estudados na área de marketing.

Em todos os trabalhos, há a tentativa de apresentar e cativar os pesquisadores em administração para o uso desta abordagem em seus trabalhos futuros. Seja fruto destes trabalhos ou não, percebe-se que nos anos seguintes, um maior número de relatos etnográficos são encontrados nas publicações nacionais (CAVEDON; FERRAZ, 2005; FLORES-PEREIRA; CAVEDON, 2009; ALCADIPANI; ROSA, 20IO; ALCADIPANI, 20IO; FERRAZ, 2OII; TURETA; ALCADIPANI, 20II). Além disso, observa-se que o tema etnografia começa a fazer parte das discussões sobre perspectivas e paradigmas em pesquisa em administração de empresas (VARGAS-HERNANDEZ, 20IO; ZACARELLI; GODOY, 2010).

Outro motivo para a baixa produção observada nos principais periódicos nacionais pode ser justificada como oriunda do próprio método etnográfico, que exige longos períodos de coleta e análise dos dados até que a publicação seja possível. Isso, frente à cobrança cada vez maior por produção científica, pode desencorajar os pesquisadores.

Mesmo assim, encontram-se trabalhos etnográficos realizados por pesquisadores brasileiros que exploram temas como cultura (CAVEDON; FERRAZ, 2005; FLORES-PEREIRA; CAVEDON, 2009; ALCADIPANI; ROSA, 20IO; FERRAZ, 20II) e estudos organizacionais e estratégias (FARIA, 2003). Esses trabalhos possuem, em comum, elementos de um bom trabalho etnográfico como autenticidade, plausibilidade nos argumentos e resultados discutidos, instigando o leitor a refletir sobre o estudo apresentado (GOLDEN-BIDDLE; LOCKE, 1993). 
Tabela 5 Grupo de artigos/autores mais citados de 200 a a 2005

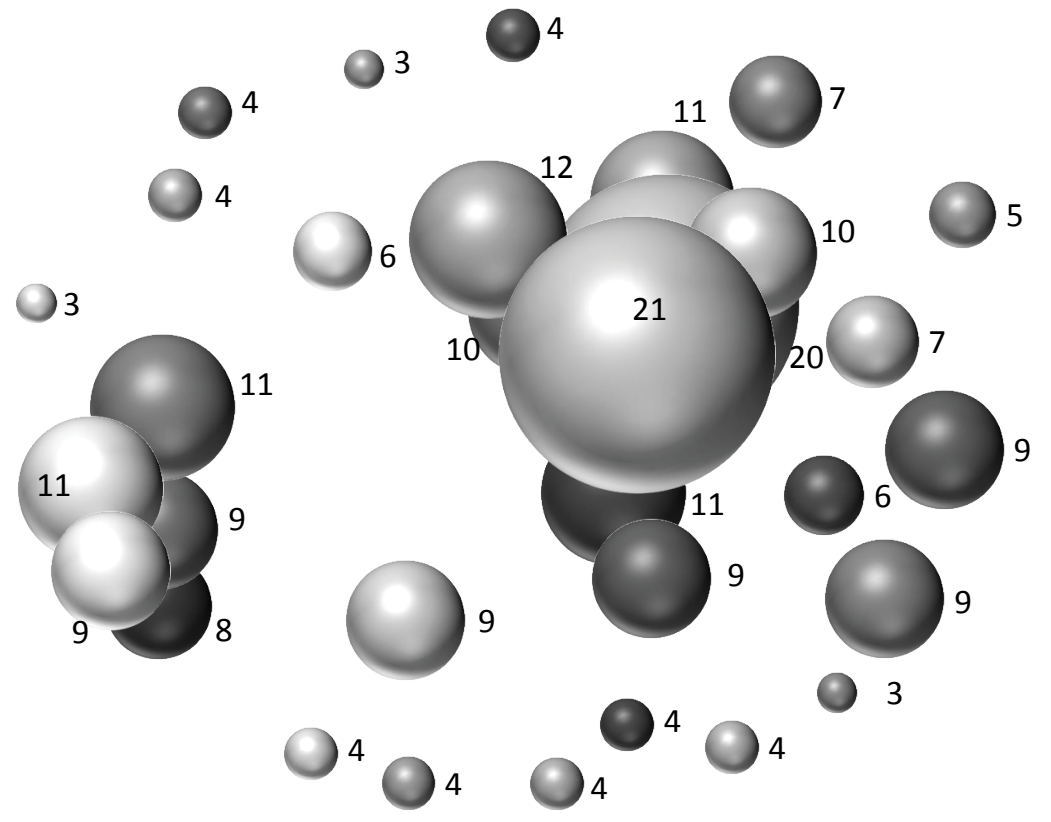

\begin{tabular}{|l|l|}
\hline Autor / ano & No de citações \\
\hline Van Maanen (1988) & 21 \\
\hline Geertz (1973) & 20 \\
\hline Van Maanen (1979) & 12 \\
\hline Clifford e Marcus (1986) & 11 \\
\hline Foucault (1980) & 11 \\
\hline Glaser e Strauss (1967) & 11 \\
\hline Schouten e McAlexander (1996) & 11 \\
\hline Marcus (1986) & 10 \\
\hline Rosen (1991) & 10 \\
\hline Alvesson e Sköldberg (2000) & 9 \\
\hline Belk, Wallendorf e Sherry Jr. (1989) & 9 \\
\hline Gerfinkel (1967) & 9 \\
\hline Giddens (1977) & 9 \\
\hline Wallendorf e Arnould (1991) & 9 \\
\hline Weick (1995) & 9 \\
\hline Belk, Sherry Jr. e Wallendorf (1988) & 8 \\
\hline
\end{tabular}




\begin{tabular}{|l|l|}
\hline Autor / ano & No de citaçöes \\
\hline Linstead (1993) & 7 \\
\hline Smircich (1983) & 6 \\
\hline Knights e Morgan (1991) & 6 \\
\hline Willmott (1993) & 5 \\
\hline Lave e Wenger (1991) & 4 \\
\hline Alvesson (1996) & 4 \\
\hline Du Gay (1996) & 4 \\
\hline Foucault (1977) & 4 \\
\hline Giddens (1976) & 4 \\
\hline Mintzberg (1973) & 4 \\
\hline Samra-Fredericks (2003) & 4 \\
\hline Sewell e Wilkinson (1992) & 4 \\
\hline Shotter (1993) & 3 \\
\hline Alvesson e Kärreman (2000) & 3 \\
\hline Du Gay e Salaman (1992) & 3 \\
\hline Law (1994)
\end{tabular}

Devido à versatilidade das metodologias qualitativas em permitir que mais de um método de coleta de dados seja empregado pelos pesquisadores, os trabalhos nacionais valem-se de técnicas mais tradicionais como a observação, as entrevistas, os diários de campo (GRAMKOW; CAVEDON, 200I; FARIA, 2003; CAVEDON; FERRAZ, 2005; PINTO; SANTOS, 2008; LEÃO; MELLO, 2009 e 2OII; FLORES-PEREIRA; CAVEDON, 2009; ALCADIPANI; ROSA, 2OIO; FERRAZ, 20II; TURETA; ALCADIPANI, 20II), por meio da coleta de dados via desenhos realizados pelos funcionários das empresas alvo dos estudos (ALCADIPANI, 20IO) ou ainda por meio de fotografias (COSTA; LEAO, 2OII)

Por fim, destaca-se que ainda são poucos os pesquisadores brasileiros que se utilizam da etnografia no entendimento dos fenômenos estudados em administração de empresas, visto que a pesquisa está concentrada no eixo sul-sudeste e que os centros acadêmicos mais atuantes são a Fundação Getúlio Vargas e a Universidade Federal do Rio Grande do Sul. 
Tabela 6 Grupo de artigos/autores mais citados de 2005 a $201 \mathrm{I}$

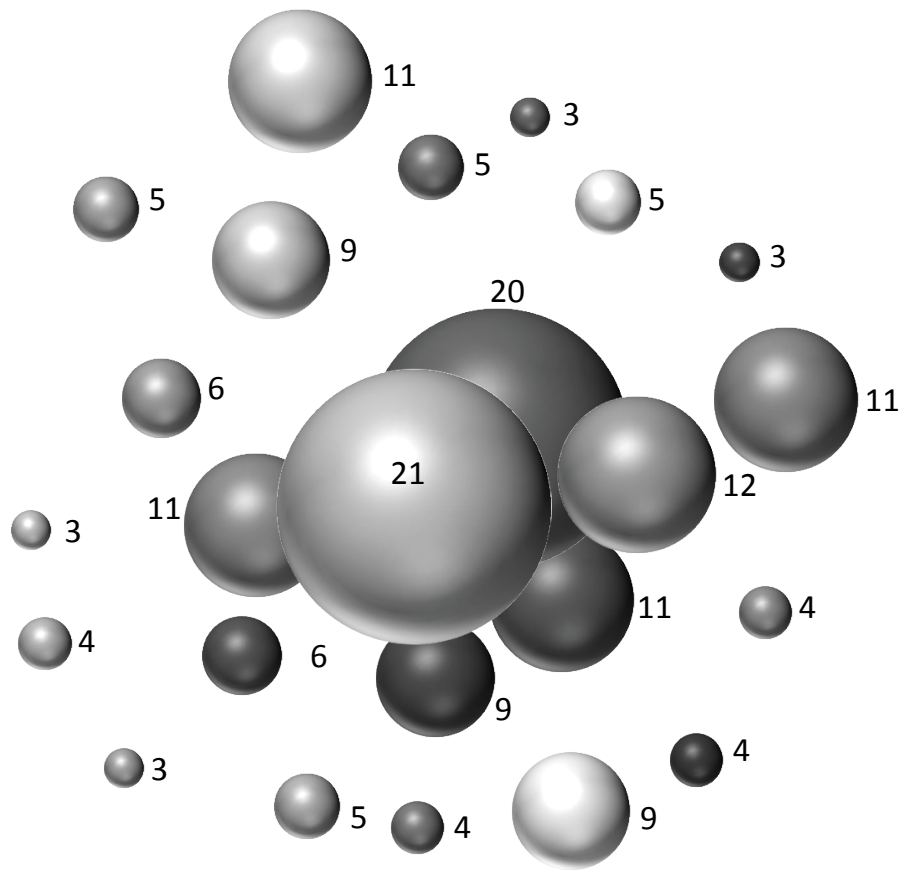

\begin{tabular}{|l|l|}
\hline Autor / ano & No de citações \\
\hline Van Maanen (1988) & 21 \\
\hline Geertz (1973) & 20 \\
\hline Van Maanen (1979) & 12 \\
\hline Clifford e Marcus (1986) & 11 \\
\hline Foucault (1980) & 11 \\
\hline Glaser e Strauss (1967) & 11 \\
\hline Schouten e McAlexander (1996) & 11 \\
\hline Alvesson e Sköldberg (2000) & 9 \\
\hline Gerfinkel (1967) & 9 \\
\hline Weick (1995) & 9 \\
\hline Jeffcutt (1994) & 6 \\
\hline Knights e Morgan (1991) & 6 \\
\hline Brown e Duguid (1991) & 5 \\
\hline Clegg, Hardy e Nord (1996) & 5 \\
\hline Collinson (1992) & 5 \\
\hline
\end{tabular}




\begin{tabular}{|l|l|}
\hline Autor / ano & No de citações \\
\hline Orr (1996) & 5 \\
\hline Alvesson (2002) & 4 \\
\hline Berger e Luckmann (1966) & 4 \\
\hline Denzin e Lincoln (1994) & 4 \\
\hline Townley (1993) & 4 \\
\hline Bechky (2003) & 3 \\
\hline Engeström e Middleton (1996) & 3 \\
\hline Gertsen, Søderberg e Torp (1998) & 3 \\
\hline Parker (2000) & 3 \\
\hline
\end{tabular}




\section{CONSIDERAÇÕES FINAIS}

O objetivo desse trabalho consiste em avaliar a evolução da etnografia por meio de uma pesquisa qualitativa mediante a análise bibliométrica de citação e cocitação, compreendendo o período de 1995 a 20II. O estudo foi realizado sob duas perspectivas: a primeira, avaliando as publicações internacionais; a segunda, avaliando as publicações nacionais.

Para fins de análise das publicações internacionais, utilizou-se a análise de citação e cocitação. Porém, para as publicações nacionais não foi possível usar essa mesma técnica devido ao fato de não se ter uma base que permitisse a extração das referências dos artigos (a base de dados Scielo não oferece esta possibilidade). A análise de cocitação está relacionada à frequência com que dois trabalhos são citados conjuntamente por outros autores e que, de certa forma, influencia os trabalhos destes (SMALL, 1973). Essa análise também permite capturar a evolução da produção da pesquisa científica e tecnológica em uma determinada área.

Os resultados dos artigos internacionais pesquisados nos principais periódicos em administração e estudos organizacionais (Human Relations, Organization Studies, Organization, etc.) mostram que as publicações de alguns autores se destacaram no período em análise e fomentaram as pesquisas posteriores. Os trabalhos de Geertz (1973), Van Maanen (1988) e Clifford e Marcus (1986) tiveram o maior número de citações por parte de pesquisadores interessados em estudos etnográficos e, portanto, são os autores que têm maior proximidade e formaram o principal grupo em relação ao tema central deste trabalho (etnografia).

No caso da análise de artigos nacionais, parece que há evidência da baixa popularidade do tema central devido ao pequeno número de publicações a partir do ano 2000. Outro fator importante observado é o foco dos pesquisadores na publicação de estudos etnográficos. Somente um trabalho científico a respeito desse tema é publicado ao ano e não necessariamente são relatos etnográficos, conforme apontam Godoy (1995b), Van Maanen (2006, 20II) e Watson (20II). Uma possível explicação para a baixa produção nacional deve-se ao método empregado (etnográfico), que 
exige longos períodos de coleta e análise dos dados até a sua publicação final. Aliado a isso, está a cobrança cada vez maior por publicações tanto no cenário nacional quanto, principalmente, no cenário internacional, que desencorajam os pesquisadores a desenvolveram pesquisas por meio dessa metodologia. No entanto, nos últimos anos, o número de publicações sobre o tema sugere que o tema passa a ser mais explorado pela comunidade acadêmica. Nesse sentido, podem ser destacados alguns pesquisadores com trabalhos relevantes no âmbito doméstico; são eles: Cavedon e Ferraz (2005), Flores-Pereira e Cavedon (2009), Alcadipani e Rosa (2010), Alcadipani (20I0), e Ferraz (20II). Do ponto de vista da pesquisa por regiões e centros acadêmicos, o eixo sul-sudeste, e, em especial, a Fundação Getúlio Vargas e a Universidade Federal do Rio Grande do Sul, destacou-se dos demais polos regionais e de geração de conhecimento.

Quanto às limitações deste trabalho, pode-se apontar a pesquisa em periódicos internacionais, que somente são considerados relevantes na área de administração e estudos organizacionais (onze periódicos) e, portanto, talvez fosse interessante ampliar essa análise para outros periódicos. Considerando a abordagem nacional, uma possibilidade seria explorar outros periódicos nas áreas das ciências sociais e antropologia e avaliar como se encontra a etnografia neste ambiente. 


\section{REFERÊNCIAS}

AGUIAR, S. M. S. P. D. A Dinâmica entre Raízes e Asas: Um estudo sobre Organizações Inovadoras. São Paulo: FGV-EAESP, 2004.

ALCADIPANI, R. Violência e masculinidade nas relações de trabalho: imagens do campo em pesquisa etnográfica. Cadernos EBAPE.BR, v. 8, n.1, p. 93-110, 2010.

ALCADIPANI, R.; ROSA, A. R. O pesquisador como o outro: Uma leitura pós-colonial do "Borat” brasileiro. Revista de Administração de Empresas, v. 50, n. 4, p. 371-382, 2010.

BACK, H. A Comparison of Operations Research and Management Science Based on Bibliographic Citations. Interfaces, v. 4, n. 2, p. 42-52, 1974.

BALESTRIN, A.; VERSCHOORE, J.R.; REYES JR., E. O Campo de Estudo sobre Redes de Cooperação. Revista de Administração Contemporânea, v. 14, n. 3, p. 458-477, 2010.

BANDEIRA-DE-MELO, R. Softwares em pesquisa qualitativa. In: GODOI, C.;

BANDEIRA-de-MELLO, R.; SILVA, A. Pesquisa Qualitativa em Estudos Organizacionais. São Paulo: Saraiva, 2006.

BLUHM, D. J.; HARMAN, W.; LEE, T. W.; MITCHELL, T. R. Qualitative Research in Management: A Decade of Progress. Journal of Management Studies, v. 48, p. 1866-1891, 2010.

BORGATTI, S.P.; EVERETT, M.G.; FREEMAN, L.C. Ucinet 6 for Windows: Software for Social Network Analysis. Harvard: Analytic Technologies, 2002.

BURRELL, G. Modernism, Post Modernism and Organizational Analysis 2: The Contribution of Michel Foucault. Organization Studies, v. 9, n. 2, p. 221-235, 1988.

CAVEDON, N. R. Fotoetnografia: a União da Fotografia com a Etnografia no Descortinamento dos Não-Ditos Organizacionais. Organizações e Sociedade, v.12, n. 35, p. 13-27, 2005.

CAVEDON, N. R.; FERRAZ, D. S. Representações sociais e estratégias em pequenos negócios. Revista de Administração de Empresas Eletrônica, v. 4, n. 1, 2005.

CLIFFORD, J.; MARCUS, G. Writing culture. The politics and poetics of ethnography. Berkeley: University of California Press, 1986.

COSTA, F. Z. N.; LEAO, A.L.M.S. Desvelamento do limiar discursivo de uma marca global em uma cultura local. Cadernos EBAPE.BR, v. 9, n. 2, p. 299-332, 2011.

DENZIN, N.; LINCOLN, Y.S. Handbook of Qualitative Research. London: Sage Publications, 1994.

FARIA, A. Pesquisa em redes estratégicas: descobertas e reflexões etnográficas. Revista de Administração de Empresas, v. 43, n. 1, p. 11-23, 2003. 
FERRAZ, D. L. S. Processo decisório e aspectos simbólicos: um estudo das culturas organizacionais da feira do livro de Porto Alegre. Organizações e Sociedade, v. 8, n. 56, p. 77-98, 2011.

FLICK, U. Introdução à Pesquisa Qualitativa. 3.ed. Porto Alegre: Artmed, 2009.

FLORES-PEREIRA, M. T.; CAVEDON, N. R. Os bastidores de um estudo etnográfico: trilhando os caminhos teórico-empíricos para desvendar as culturas organizacionais de uma livraria de shopping center. Cadernos EBAPE.BR, v. 7, n. 1, p. 153-168, 2009.

FOUCAULT, M. Power/Knowledge: Selected Interviews \& Other Writings. New York: Pantheon Books, 1980.

GEERTZ, C. The Interpretation of cultures. New York: Basic Books, 1973.

GLASER, B.G.; STRAUSS, A.L. The discovery of grounded theory: strategies for qualitative research. New Jersey: Transaction Publishers, 1967.

GODOY, S. A. Introdução à Pesquisa Qualitativa e Suas Possibilidades. Revista de Administração de Empresas, v. 35, n. 2, p. 57-63, 1995a.

GODOY, S.A. Pesquisa Qualitativa - Tipos Fundamentais. Revista de Administração de Empresas, v. 35, n. 3, p. 20-29, 1995 b.

GODOY, S.A. Pesquisa Qualitativa e Sua Utilização em Administração de Empresas. Revista de Administração de Empresas, v. 35, n. 4, p. 20-29, 1995c.

GOLDEN-BIDDLE, K.; LOCKE, K. Appealing Work: an Investigation of How Ethnographic Texts Convince. Organization Science, v. 4, n. 4, p. 595-616, 1993.

GRAMKOW, F.; CAVEDON, N. R. As bancas de especiarias do Mercado Público de Porto Alegre e suas Estratégias. Organizações e Sociedade, v.8, n. 2, p. 161-174, 2001.

KUNDA, G. Engineering Culture: Control and Commitment in a Hight Tech Corporation. Philadelphia: Temple University Press, 2006.

LEAO, A.L.M.S.; MELLO, S. C.B. Atividades Marcárias na Vida Cotidiana dos Consumidores: Descoberta de uma Nova Forma de se Pensar as Marcas? Revista de Administração Contemporânea, v. 13, n. 1, p. 93-116, 2009.

LEAO, A.L.M.S.; MELLO, S.C.B. Stigmata: como as marcas são usadas para marcar os consumidores. Cadernos EBAPE.BR, v. 9, n. 1, p. 22- 36, 2011.

LIM, A.; MA, H.; WEN, Q.; XU, Z.; CHEANG. B. Distinguishing citation quality for journal impact assessment. Communications of the ACM, v. 52, n. 8, p. 111-116, 2009.

MARCUS, G.E. Contemporary problems of ethnography in the modern world system. In: CLIFFORD, J.C.; GE, M. (Eds.). Writing Culture. Berkeley and Los Angeles: University of California. Press, 1986.

MASCARENHAS, A.O. Etnografia e Cultura Organizacional: Uma Contribuição da Antropologia à Administração de Empresas. Revista de Administração de Empresas, v. 42, n. 2, p. 88-94, 2002. 
MALINOWSKI, B. Baloma: Spirits of the dead in the Trobriand Islands. Journal of the Royal Anthropological Institute, v. 46, p. 354-430, 1916.

OKUBO, Y. Bibliometric Indicators and analysis of research systems: methods and examples Paris: OECD, 1997, 69p (STI Working Papers, 1997/1).

PINTO, M. R.; SANTOS, L.L.S. Em busca de uma trilha interpretativista para pesquisa do consumidor: uma proposta baseada na fenomenologia, na etnografia e na grounded theory. Revista de Administração de Empresas Eletrônica, v. 7, n. 2, 2008.

ROSEN, M. Coming to terms with the field: understanding and doing organizational ethnography. Journal of Management Studies, v. 28, n.1, p. 1-24, 1991.

SCHILDT, H.A. SITKIS: Software for Bibliometric Data Management and Analysis. v. 6.1. Helsinki: Institute of Strategy and International Business. Disponível em: <www. hut.fi/ hschildt/sitkis>. Acesso em: 17/09/2002.

SCHOUTEN, J.W.; MCALEXANDER, J.H. Subcultures of Consumption: An Ethnography of the New Bikers Subcultures of Consumption: An Ethnography of the New Bikers. The Journal of Consumer Research, v. 22, n. 1, p. 43-61, 1996.

SEGLEN, P. The skewness of science. Journal of the American Society for Information Science and Technology, v. 43, n. 9, p. 628-638, 1992.

SMALL, H.G. Co-citation in the Scientific Literature: A New Measure of the Relationship Between Documents. Journal of American Society for Information Science, v. 24, n. 4, p. 265-269, 1973.

STRAUSS, A. L. Qualitative analysis for social scientists. Cambridge: Cambridge University Press, 2003.

TURETA, C.; ALCADIPANI, R. Entre o Observador e o Integrante da Escola de Samba: os Não-Humanos e as Transformações Durante uma Pesquisa de Campo. Revista de Administração Contemporânea, v. 15, n. 2, p. 209-227, 2011.

USDISKEN, B.; PASADEOS Y. Organizational Analysis in North America and Europe: A Comparison of Co-citation Networks. Organization Studies, v. 16, n. 3, p. 503-526, 1995.

VAN MAANEN, J. The fact of fiction in organizational ethnography. Administrative Science Quarterly, v. 24, n. 4, p. 539-550, 1979.

VAN MAANEN, J. Tales of the Field: On Writing Ethnography. Chicago, IL: University of Chicago Press, p. 2-10, 1988.

VAN MAANEN, J. Ethnography then and now: Qualitative Research in Organizations and Management. An International Journal, v. 1, n. 1, p. 13-21, 2006.

VAN MAANEN, J. Ethnography as Work: Some Rules of Engagement. Journal of Management Studies, v. 48, n. 1, p. 218-234, 2011.

VARGAS-HERNANDEZ J. G. Contínuo pendular de la investigación en la administración internacional de las organizaciones: del positivismo funcionalista a la investigación cualitativa y etnográfica. Cadernos EBAPE.BR, v. 8, n. 1, p. 112-132, 2010. 
WATSON, T. J. Ethnography, Reality, and Truth: The Vital Need for Studies of 'How Things Work' in Organizations and Management. Journal of Management Studies, v. 48, n. 1, p. 202-217, 2011.

WEITZMAN, E. Software and Qualitative Research. In: DENZIN, N.; LINCOLN, Y. Handbook of Qualitative Research. London: Sage Publications, 2000.

WHYTE, W. Street Corner Society: The Social Structure of an Italian Slum. Chicago: The University of Chicago Press, 1943.

WILLIAMS, M. Avatar watching: participant observation in graphical online environments. Qualitative Research, v. 7, n. 1, p. 5-24, 2007.

YIN, R. K. Case Studiy Research - Design and Methods. 3.ed., v. 5, Newbury Park: Sage Publications, 2002.

ZACARELLI, L.M.;GODOY, A.S. Perspectivas do uso de diários nas pesquisas em organizações. Cadernos EBAPE.BR, v. 8, n. 3, p. 550-563, 2010. 


\section{DADOS DOS AUTORES}

CHEN YEN-TSANG ${ }^{\star}$ yentsang.chen@gmail.com

Doutorando em Administração de Empresas pela Fundação Getúlio Vargas/ EAESP Instituição de vinculação: Fundação Getúlio Vargas - Escola de Administração de Empresas de São Paulo.

São Paulo/SP - Brasil

Áreas de interesse em pesquisa: Gestão de operações, Estratégia Empresarial, Competitividade, Relacionamento Comprador/Fornecedor, Capital Social e Guanxi.

${ }^{\star}$ Rua Itapeva, $4328^{\circ}$ andar-Departamento Gestão de Operações

Bela Vista São Paulo/SP CEP: 01332-000

RONALDO GOMES DULTRA-DE-LIMA ronaldo.lima@gvmail.br Doutorando em Administração de Empresas pela Fundação Getúlio Vargas/ EAESP Instituição de vinculação: Fundação Getúlio Vargas - Escola de Administração de Empresas de São Paulo e Professor da Universidade Presbiteriana Mackenzie.

São Paulo/SP - Brasil

Áreas de interesse em pesquisa: Gestão de Operações e Competitividade, Gestão de Projetos e Controle Gerencial.

\section{KARINA PRETTO karina.pretto@gvmail.br}

Doutoranda em Administração de Empresas pela Fundação Getúlio Vargas/ EAESP Instituição de vinculação: Fundação Getúlio Vargas - Escola de Administração de Empresas de São Paulo.

São Paulo/SP - Brasil

Áreas de interesse em pesquisa: Gestão de Operações e Competitividade, Estratégia Empresarial e Análise Quantitativa. 